\title{
Author Index Volume 25 (2015)
}

Abolghasemi, V., see Ferdowsi, S.

$1(2015) 1440008$

Abou Zeid, E. \& Chau, T., Electrode fusion for the prediction of self-initiated fine movements from single-trial readiness potentials

Amirfattahi, R., see Vahabi, Z.

Arbuthnott, G. W., see Carrillo-Reid, L.

Arica, N., see Aydin, S.

Aydin, S., Arica, N., Ergul, E. \& Tan, O., Classification of obsessive compulsive disorder by EEG complexity and hemispheric dependency measurements

Bandarabadi, M., Rasekhi, J., Teixeira, C. A., Netoff, T. I., Parhi, K. K. \& Dourado, A., Early seizure detection using neuronal potential similarity: a generalized low-complexity and robust measure

$4(2015) 1550014$

6 (2015) 1550028

7 (2015) 1550026

3 (2015) 1550010

3 (2015) 1550010

Bellistri, E., Sartori, I., Pelliccia, V., Francione, S., Cardinale, F., de Curtis, M. \& Gnatkovsky, V., Fast activity evoked by intracranial $50 \mathrm{~Hz}$ electrical stimulation as a marker of the epileptogenic zone

Berdiñas, B. G., see Castillo, E.

Bonanno, L., see Morabito, F. C.

Boon, P., see Grimonprez, A.

Bourbakis, N., see Michalopoulos, K.

Bramanti, A., see Morabito, F. C.

Bramanti, P., see Morabito, F. C.

Cadaveira, F., see Correas, A.

Campolo, M., see Morabito, F. C.

Cao, W., see Luo, C.

Cardinale, F., see Bellistri, E.

Carrillo-Reid, L., Lopez-Huerta, V. G., Garcia-Munoz, M., Theiss, S. \& Arbuthnott, G.

W., Cell assembly signatures defined by short-term synaptic plasticity in cortical networks

Castillo, E., Peteiro-Barral, D., Berdiñas, B. G. \& Fontenla-Romero, O., Distributed one-class support vector machine

Cedrón, F., see Mesejo, P.

Chau, T., see Abou Zeid, E.

Chen, Y.-Y., see Su, F.

Chersi, F., see Donnarumma, F.

Chira, C., see Villar, J. R.

Chyzhyk, D., Graña, M., Öngür, D. \& Shinn, A. K., Discrimination of schizophrenia auditory hallucinators by machine learning of resting-state functional MRI

Cichocki, A., see Jin, J.

Claus, S. P., see Geertsema, E. E.

7 (2015) 1550026

$5(2015) 1550022$

7 (2015) 1550029

$2(2015) 1550005$

8 (2015) 1550034

8 (2015) 1550041

2 (2015) 1550005

2 (2015) 1550005

3 (2015) 1550008

2 (2015) 1550005

6 (2015) 1550027

5 (2015) 1550022

7 (2015) 1550029

4 (2015) 1550012

4 (2015) 1550014

1 (2015) 1450030

6 (2015) 1550017

4 (2015) 1450036

3 (2015) 1550007

3 (2015) 1550011

5 (2015) 1550015 
Correas, A., Rodriguez Holguín, S., Cuesta, P., López-Caneda, E., García-Moreno, L. M., Cadaveira, F. \& Maestú, F., Exploratory analysis of power spectrum and functional connectivity during resting state in young binge drinkers: A MEG study

Cuesta, P., see Correas, A.

da Cruz Lotufo, C. M., see Mari, J. F.

de Curtis, M., see Bellistri, E.

Delbeke, J., see Grimonprez, A.

Deleforge, A., Forbes, F. \& Horaud, R., Acoustic space learning for sound-source separation and localization on binaural manifolds

Deng, B., see Su, F.

de Salvo, S., see Morabito, F. C.

De Taeye, L., see Grimonprez, A.

Destro-Filho, J.-B., see Mari, J. F.

Dittrich, P., see Gruenert, G.

do Carmo Nicoletti, M., see Mari, J. F.

Donnarumma, F., Prevete, R., Chersi, F. \& Pezzulo, G., A programmer-interpreter neural network architecture for prefrontal cognitive control

Donos, C., Dümpelmann, M. \& Schulze-Bonhage, A., Early seizure detection algorithm based on intracranial EEG and random forest classification

Dourado, A., see Bandarabadi, M.

Dreyfus, G., see Houmani, N.

Dümpelmann, M., see Donos, C.

Eikenberry, S. E. \& Marmarelis, V. Z., Principal dynamic mode analysis of the Hodgkin-Huxley equations

Ergul, E., see Aydin, S.

Escuela, G., see Gruenert, G.

Etxeberria-Agiriano, I., see Suberbiola, A.

Ferdowsi, S., Sanei, S. \& Abolghasemi, V., A predictive modeling approach to analyze data in EEG-fMRI experiments

Fernández-Blanco, E., see Mesejo, P.

Fontenla-Romero, O., see Castillo, E.

Forbes, F., see Deleforge, A.

Francione, S., see Bellistri, E.

García-Moreno, L. M., see Correas, A.

Garcia-Munoz, M., see Carrillo-Reid, L.

Geertsema, E. E., Visser, G. H., Velis, D. N., Claus, S. P., Zijlmans, M. \& Kalitzin, S. N., Automated seizure onset zone approximation based on nonharmonic high-frequency oscillations in human interictal intracranial EEGs

Ghassemi, F., see Vahabi, Z.

Gizynski, K., see Gruenert, G.

Gnatkovsky, V., see Bellistri, E.

González, S., see Villar, J. R.

Gorecki, J., see Gruenert, G.

Graña, M., see Suberbiola, A.

Graña, M., see Chyzhyk, D.

Grimonprez, A., Raedt, R., De Taeye, L., Larsen, L. E., Delbeke, J., Boon, P. \& Vonck, K., A preclinical study of laryngeal motor-evoked potentials as a marker vagus nerve activation
3 (2015) 1550008

3 (2015) 1550008

8 (2015) 1550033

5 (2015) 1550022

8 (2015) 1550034

1 (2015) 1440003

1 (2015) 1450030

2 (2015) 1550005

8 (2015) 1550034

8 (2015) 1550033

7 (2015) 1450032

8 (2015) 1550033

6 (2015) 1550017

5 (2015) 1550023

5 (2015) 1550019

8 (2015) 1550032

5 (2015) 1550023

2 (2015) 1550001

3 (2015) 1550010

7 (2015) 1450032

3 (2015) 1550009

1 (2015) 1440008

4 (2015) 1550012

7 (2015) 1550029

1 (2015) 1440003

5 (2015) 1550022

3 (2015) 1550008

7 (2015) 1550026

5 (2015) 1550015

6 (2015) 1550028

7 (2015) 1450032

5 (2015) 1550022

4 (2015) 1450036

7 (2015) 1450032

3 (2015) 1550009

3 (2015) 1550007

8 (2015) 1550034 
Gruenert, G., Gizynski, K., Escuela, G., Ibrahim, B., Gorecki, J. \& Dittrich, P., Understanding networks of computing chemical droplet neurons based on information flow

Gu, Z., see Liu, K.

Helling, R. M., Koppert, M. M. J., Visser, G. H. \& Kalitzin, S. N., Gap junctions as common cause of high-frequency oscillations and epileptic seizures in a computational cascade of neuronal mass and compartmental modeling

Horaud, R., see Deleforge, A.

Houmani, N., Dreyfus, G. \& Vialatte, F. B., Epoch-based entropy for early screening of Alzheimer's disease

Hsu, W.-Y., Assembling a multi-feature EEG classifier for left-right motor imagery data using wavelet-based fuzzy approximate entropy for improved accuracy

Huang, B., see Zhang, D.

Huang, Y., see Luo, C.

Ibáñez, O., see Mesejo, P.

Ibrahim, B., see Gruenert, G.

Jeavons, P., see $\mathrm{Xu}, \mathrm{L}$.

Jin, J., Sellers, E. W., Zhou, S., Zhang, Y., Wang, X. \& Cichocki, A., A P300

brain-computer interface based on a modification of the mismatch negativity paradigm

Jung, M., see Lin, C.-L.

Jung, T.-P., see Lin, C.-L.

Kalitzin, S. N., see Geertsema, E. E.

Kalitzin, S. N., see Helling, R. M.

Kälviäinen, R., see Kimiskidis, V. K.

Kimiskidis, V. K., Koutlis, C., Tsimpiris, A., Kälviäinen, R., Ryvlin, P. \& Kugiumtzis, D., Transcranial magnetic stimulation combined with EEG reveals covert states of elevated excitability in the human epileptic brain

Kimiskidis, V. K., see Kugiumtzis, D.

Koppert, M. M. J., see Helling, R. M.

Koutlis, C., see Kimiskidis, V. K.

Kugiumtzis, D. \& Kimiskidis, V. K., Direct causal networks for the study of transcranial magnetic stimulation effects on focal epileptiform discharges

Kugiumtzis, D., see Kimiskidis, V. K.

Labate, D., see Morabito, F. C.

Längkvist, M. \& Loutfi, A., Learning feature representations with a cost-relevant sparse autoencoder

Larsen, L. E., see Grimonprez, A.

Li, H.-Y., see Su, F.

Li, S., see Liu, Y.

Li, S., see Zhang, D.

Li, X., see Yuan, S.

Li, Y., see Liu, K.

Lin, C.-L., Jung, M., Wu, Y. C., She, H.-C. \& Jung, T.-P., Neural correlates of mathematical problem solving

Liu, C., see Su, F.

Liu, K., Yu, Z. L., Wu, W., Gu, Z. \& Li, Y., STRAPS: A fully data-driven spatio-temporally regularized algorithm for M/EEG patch source imaging

Liu, Y., Ning, Y., Li, S., Zhou, P., Rymer, W. Z. \& Zhang, Y., Three-dimensional innervation zone imaging from multi-channel surface EMG recordings
7 (2015) 1450032

$4(2015) 1550016$

6 (2015) 1550021

1 (2015) 1440003

8 (2015) 1550032

8 (2015) 1550037

7 (2015) 1550030

6 (2015) 1550027

4 (2015) 1550012

7 (2015) 1450032

7 (2015) 1550025

3 (2015) 1550011

2 (2015) 1550004

2 (2015) 1550004

5 (2015) 1550015

6 (2015) 1550021

5 (2015) 1550018

5 (2015) 1550018

5 (2015) 1550006

6 (2015) 1550021

5 (2015) 1550018

5 (2015) 1550006

5 (2015) 1550018

2 (2015) 1550005

1 (2015) 1450034

8 (2015) 1550034

1 (2015) 1450030

6 (2015) 1550024

7 (2015) 1550030

2 (2015) 1550003

4 (2015) 1550016

2 (2015) 1550004

1 (2015) 1450030

4 (2015) 1550016

6 (2015) 1550024 
Liu, Y., Zhao, Q. \& Zhang, L., Uncorrelated multiway discriminant analysis for motor imagery EEG classification

López-Caneda, E., see Correas, A.

Lopez-Gordo, M. A., Sánchez-Morillo, D. \& Van Gerven, M. A. J., Spreading codes enables the blind estimation of the hemodynamic response with short-events sequences

Lopez-Guede, J. M., see Suberbiola, A.

Lopez-Huerta, V. G., see Carrillo-Reid, L.

Loutfi, A., see Längkvist, M.

Luo, C., Zhang, Y., Cao, W., Huang, Y., Yang, F., Wang, J., Tu, S., Wang, X. \& Yao, D., Altered structural and functional feature of striato-cortical circuit in benign epilepsy with centrotemporal spikes

Ma, S., see Wang, H.

Maestú, F., see Correas, A.

Mari, J. F., Saito, J. H., Neves, A. F., da Cruz Lotufo, C. M., Destro-Filho, J.-B. \& do Carmo Nicoletti, M., Quantitative analysis of rat dorsal root ganglion neurons cultured on microelectrode arrays based on fluorescence microscopy image processing

Marmarelis, V. Z., see Eikenberry, S. E.

Marra, A., see Morabito, F. C.

Mesejo, P., O. IbáñezFernández-Blanco, E., Cedrón, F., Pazos, A. \& Porto-Pazos, A. B., Artificial Neuron-Glia networks learning approach based on cooperative coevolution

Michalopoulos, K. \& Bourbakis, N., Combining EEG microstates with fMRI structural features for modeling brain activity

Morabito, F. C., Campolo, M., Labate, D., Morabito, G., Bonanno, L., Bramanti, A., de Salvo, S., Marra, A. \& Bramanti, P., A longitudinal EEG study of Alzheimer's disease progression based on a complex network approach

Morabito, G., see Morabito, F. C.

Netoff, T. I., see Bandarabadi, M.

Neves, A. F., see Mari, J. F.

Ning, Y., see Liu, Y.

Öngür, D., see Chyzhyk, D.

Parhi, K. K., see Bandarabadi, M.

Pazos, A., see Mesejo, P.

Pelliccia, V., see Bellistri, E.

Peteiro-Barral, D., see Castillo, E.

Pezzulo, G., see Donnarumma, F.

Porto-Pazos, A. B., see Mesejo, P.

Prevete, R., see Donnarumma, F.

Raedt, R., see Grimonprez, A.

Rasekhi, J., see Bandarabadi, M.

Rodriguez Holguín, S., see Correas, A.

Rymer, W. Z., see Liu, Y.

Ryvlin, P., see Kimiskidis, V. K.

Saito, J. H., see Mari, J. F.

Sánchez-Morillo, D., see Lopez-Gordo, M. A.

Sanei, S., see Ferdowsi, S.

Sartori, I., see Bellistri, E.

Schulze-Bonhage, A., see Donos, C.

Sedano, J., see Villar, J. R.

Sellers, E. W., see Jin, J.

4 (2015) 1550013

3 (2015) 1550008

1 (2015) 1450035

3 (2015) 1550009

7 (2015) 1550026

1 (2015) 1450034

6 (2015) 1550027

2 (2015) 1550002

3 (2015) 1550008

8 (2015) 1550033

2 (2015) 1550001

2 (2015) 1550005

4 (2015) 1550012

8 (2015) 1550041

2 (2015) 1550005

2 (2015) 1550005

5 (2015) 1550019

8 (2015) 1550033

6 (2015) 1550024

3 (2015) 1550007

5 (2015) 1550019

4 (2015) 1550012

5 (2015) 1550022

7 (2015) 1550029

6 (2015) 1550017

4 (2015) 1550012

6 (2015) 1550017

8 (2015) 1550034

5 (2015) 1550019

3 (2015) 1550008

6 (2015) 1550024

5 (2015) 1550018

8 (2015) 1550033

1 (2015) 1450035

1 (2015) 1440008

5 (2015) 1550022

5 (2015) 1550023

4 (2015) 1450036

3 (2015) 1550011 
Shayegh, F., see Vahabi, Z.

6 (2015) 1550028

She, H.-C., see Lin, C.-L.

2 (2015) 1550004

Shi, T., see Wang, H.

Shinn, A. K., see Chyzhyk, D.

Su, F., Wang, J., Deng, B., Wei, X.-L., Chen, Y.-Y., Liu, C. \& Li, H.-Y., Adaptive

2 (2015) 1550002

3 (2015) 1550007

control of Parkinson's state based on a nonlinear computational model with unknown parameters

Suberbiola, A., Zulueta, E., Lopez-Guede, J. M., Etxeberria-Agiriano, I. \& Graña, M., Arm orthosis/prosthesis movement control based on surface EMG signal extraction

Tan, O., see Aydin, S.

Teixeira, C. A., see Bandarabadi, M.

Theiss, S., see Carrillo-Reid, L.

Trejo-Gabriel-Galan, J. M., see Villar, J. R.

Tsimpiris, A., see Kimiskidis, V. K.

Tsuda, I., see Wang, R.

Tu, S., see Luo, C.

Vahabi, Z., Amirfattahi, R., Shayegh, F. \& Ghassemi, F., Online epileptic seizure prediction using wavelet-based bi-phase correlation of electrical signals tomography

Van Gerven, M. A. J., see Lopez-Gordo, M. A.

Velis, D. N., see Geertsema, E. E.

Vialatte, F. B., see Houmani, N.

Villar, J. R., González, S., Sedano, J., Chira, C. \& Trejo-Gabriel-Galan, J. M., Improving human activity recognition and its application in early stroke diagnosis

Visser, G. H., see Geertsema, E. E.

Visser, G. H., see Helling, R. M.

Vonck, K., see Grimonprez, A.

Wang, F., see Wang, H.

Wang, H., Zhang, C., Shi, T., Wang, F. \& Ma, S., Real-time EEG-based detection of fatigue driving danger for accident prediction

Wang, J., see Luo, C.

Wang, J., see $\mathrm{Su}, \mathrm{F}$.

Wang, J., see Yuan, S.

Wang, R., Tsuda, I. \& Zhang, Z., A new work mechanism on neuronal activity

Wang, X., see Jin, J.

Wang, X., see Luo, C.

Wei, X.-L., see $\mathrm{Su}, \mathrm{F}$.

$\mathrm{Wu}$, Q., see Yuan, S.

$\mathrm{Wu}, \mathrm{W}$., see Liu, K.

Wu, W., see Zhang, D.

Wu, Y. C., see Lin, C.-L.

$\mathrm{Xu}, \mathrm{L} . \&$ Jeavons, P., Simple algorithms for distributed leader election in anonymous synchronous rings and complete networks inspired by neural development in fruit flies

Yang, F., see Luo, C.

Yao, D., see Luo, C.

Yu, Z. L., see Liu, K.

Yuan, Q., see Yuan, S.

Yuan, S., Zhou, W., Yuan, Q., Li, X., Wu, Q., Zhao, X. \& Wang, J., Kernel collaborative representation-based automatic seizure detection in intracranial EEG

Yuan, S., see Zhang, Y.

$1(2015) 1450030$

3 (2015) 1550009

3 (2015) 1550010

5 (2015) 1550019

7 (2015) 1550026

4 (2015) 1450036

5 (2015) 1550018

3 (2015) 1450037

6 (2015) 1550027

6 (2015) 1550028

1 (2015) 1450035

5 (2015) 1550015

8 (2015) 1550032

4 (2015) 1450036

5 (2015) 1550015

6 (2015) 1550021

8 (2015) 1550034

2 (2015) 1550002

2 (2015) 1550002

6 (2015) 1550027

1 (2015) 1450030

2 (2015) 1550003

3 (2015) 1450037

3 (2015) 1550011

6 (2015) 1550027

1 (2015) 1450030

2 (2015) 1550003

4 (2015) 1550016

7 (2015) 1550030

2 (2015) 1550004

7 (2015) 1550025

6 (2015) 1550027

6 (2015) 1550027

4 (2015) 1550016

2 (2015) 1550003

2 (2015) 1550003

6 (2015) 1550020 
Zhang, C., see Wang, H.

$2(2015) 1550002$

Zhang, D., Huang, B., Wu, W. \& Li, S., An idle-state detection algorithm for

SSVEP-based brain-computer interfaces using a maximum evoked response spatial

filter

7 (2015) 1550030

Zhang, D., Zhang, Q. \& Zhu, X., Exploring a type of central pattern generator based on

Hindmarsh-Rose model: From theory to application

Zhang, L., see Liu, Y.

Zhang, Q., see Zhang, D.

Zhang, Y., Zhou, W. \& Yuan, S., Multifractal analysis and relevance vector machine-based automatic seizure detection in intracranial EEG

Zhang, Y., see Jin, J.

Zhang, Y., see Liu, Y.

Zhang, Y., see Luo, C.

Zhang, Z., see Wang, R.

1 (2015) 1450028

4 (2015) 1550013

1 (2015) 1450028

Zhao, Q., see Liu, Y.

Zhao, X., see Yuan, S.

6 (2015) 1550020

3 (2015) 1550011

6 (2015) 1550024

6 (2015) 1550027

3 (2015) 1450037

4 (2015) 1550013

Zhou, P., see Liu, Y.

2 (2015) 1550003

Zhou, S., see Jin, J.

6 (2015) 1550024

Zhou, W., see Yuan, S.

3 (2015) 1550011

Zhou, W., see Zhang, Y.

2 (2015) 1550003

6 (2015) 1550020

Zhu, X., see Zhang, D.

1 (2015) 1450028

Zijlmans, M., see Geertsema, E. E.

5 (2015) 1550015

Zulueta, E., see Suberbiola, A.

3 (2015) 1550009 